\title{
Original Article \\ Role of radionuclide scintigraphy in the detection of parathyroid adenoma
}

\author{
Singh N, Krishna BA \\ Department of Nuclear Medicine, P. D. Hinduja National Hospital and MRC, Mumbai, India
}

Correspondence to: Dr. Natasha Singh, Email: drnatasha_singh@yahoo.com

\begin{abstract}
BACKGROUND: Preoperative detection of parathyroid adenoma is a diagnostic challenge. The sonography and computerized tomography (CT) scan demonstrate high sensitivity but low specificity. The advent of radionuclide scanning technique has enhanced the specificity in this context. AIM: We undertook a study to assess the role of radionuclide scanning in suspected cases of parathyroid adenomas. MATERIALS AND METHODS: Totally 28 cases were incorporated in the study. The suspicion was raised either due to raised PTH levels or recurrent calcinosis. Most of these patients had estimation of calcium done as a routine or specific investigation. The parathyroid scan was performed using either of the two techniques - Dual isotope subtraction or Sestamibi washout technique. We also used the recent approach of fusion imaging (CT + tomographic nuclear images) in selected cases. RESULTS: There were 16 true positive, 10 true negative, 1 false negative and 1 equivocal scan findings. The findings were compared with sonography, CT Scan and PTH values. The true positive yield in our study was 57\%, true negative $35 \%$ and the overall sensitivity and specificity was found to be $94 \%$ and $100 \%$ respectively. CONCLUSION: We conclude that parathyroid scintigraphy is a reliable and sensitive technique in the preoperative detection of parathyroid adenomas and should be the first choice of imaging modality in suspicion of parathyroid adenoma.
\end{abstract}

Key words: Highest sensitivity and specificity, parathyroid adenoma, preoperative detection, radionuclide scintigraphy

\section{Introduction}

Parathyroid adenoma is one of the major causes of primary hyperparathyroidism, others being parathyroid hyperplasia and rarely carcinoma (ranging in incidence from $0.3 \%$ to $4.6 \%$ ). About $10-20 \%$ of all the patients with primary hyperpara-thyroidism present with multiple gland disease, that is, presence of more than one pathological parathyroid gland. ${ }^{[1,2]}$

Over the last decades, the clinical manifestations have changed from the more obvious classical triad described by Castleman as "renal stones, painful bones and abdominal groans" to patients with vague neuromuscular and behavioural symptoms or even without any symptoms, the diagnosis being suspected because of the finding of hypercalcemia. The symptoms are the direct effect of excess PTH secretion and the induced hypercalcemia. Hyperparathyroidism is caused by a solitary adenoma in $80 \%$ to $85 \%$ of the cases, involving one or part of one parathyroid gland. They are often ellipsoidal, spherical or oval, with their weight varying most frequently within 300 to $1000 \mathrm{mg}$ and with the predominance of 'chief cells'. The remaining $15 \%$ to $20 \%$ of the cause of hyperparathyroidism is made up by predominantly chief cell hyperplasia. The glands are affected asymmetrically and enlarged glands coexist with small or normal appearing glands. Microscopic hyperplasia, often focal, affects all glands irrespective of size. 
Surgery remains the mainstay of corrective treatment for parathyroid adenomas. ${ }^{[1,3]}$ Apart from being able to differentiate between primary and secondary hyperparathyroidism, the preoperative detection of parathyroid adenomas still remains a diagnostic challenge in spite of the various imaging modalities available to us today. The intraoperative differentiation between an adenoma, hyperplasia and a single adenoma from multiple gland disease is complicated by the asymmetrical location of the parathyroid glands, some of which may be diseased when not enlarged (Kaplan $e t$ al, 1992).

Currently, sonography and computerized tomography (CT) scan are used preoperatively for their detection.

Both these modalities lack specificity ${ }^{[1,3]}$ and hence, intraoperative dye injection technique continued to be used as a reliable method. However, the advent of isotope technique has made the preoperative detection of parathyroid adenomas not only easy but also specific.

\section{Aim}

We undertook a study to assess the role of radionuclide scintigraphy in the preoperative detection of parathyroid adenomas.

\section{Materials and Methods}

A total of 28 patients within the period from February 2004 - March 2005 were included in this retrospective study. Only those patients were included in whom suspicion was raised either on symptomatology, biochemical investigations or other imaging modalities like sonography or CT scan. Isotope imaging was performed to either confirm or rule out the same. Among the host of presenting symptoms varying from non-specific symptoms like generalized weakness to specific symptoms like renal stones or backache - it was interesting to note that nephrocalcinosis formed the major group of clinical presentation [Table 1]. Most of these patients had an estimation of calcium either as a part of routine study or specifically in patients with nephrocalcinosis.

In our study, $53.57 \%$ patients had high calcium levels. In view of the raised calcium levels, the PTH was estimated followed by either sonography or CT scan.

In the early 1990's, the first technique used for imaging was referred to as the DUAL ISOTOPE subtraction technique. The second technique came into vogue in 1995, which was referred to as the WASHOUT

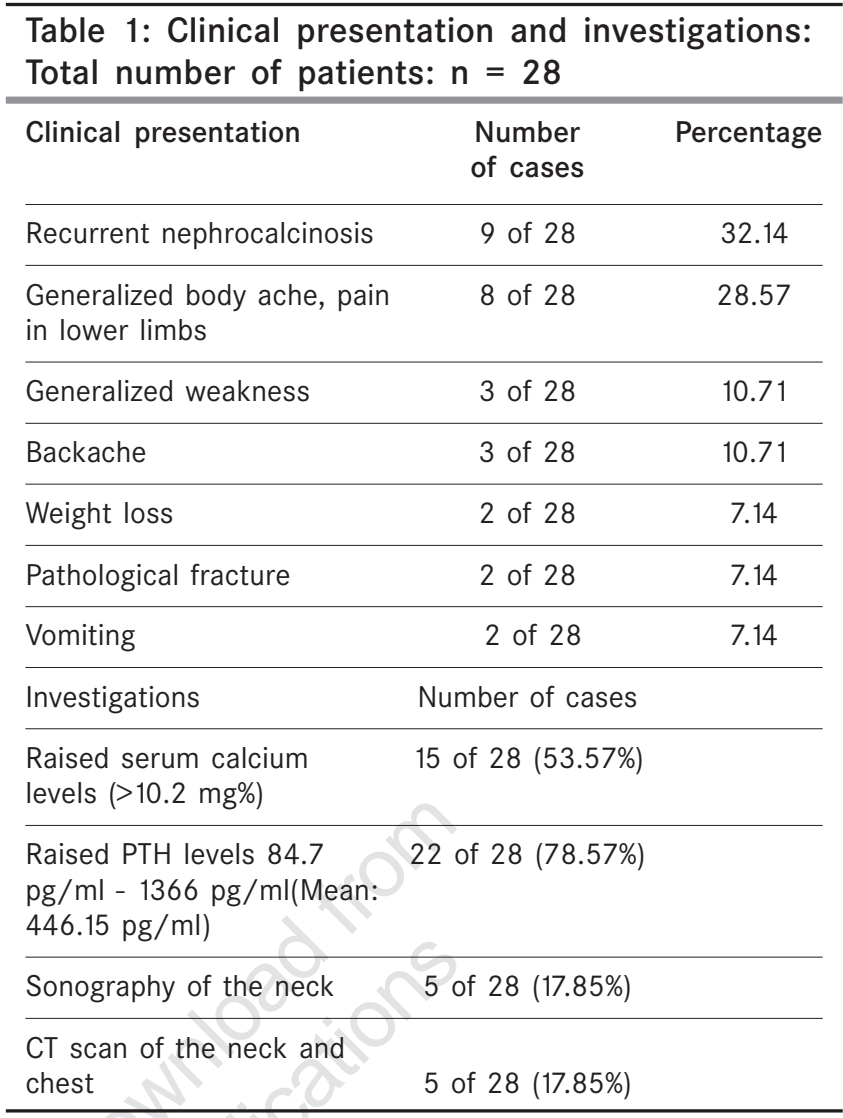

technique. We used both these techniques of scanning, each based on different concepts of imaging. ${ }^{[1,4,5]}$

\section{Imaging protocols}

The patient is in the supine position, with the neck slightly extended beneath the detector and the head is immobilized. This positioning ensures that the thyroid gland will be in the upper part of the field, thus allowing the upper 6 to $8 \mathrm{~cm}$ of the mediastinum to be visualized.

\section{Dual isotope subtraction technique}

Technetium $\left({ }^{99 \mathrm{~m}} \mathrm{Tc}\right)$ and Thallium $\left({ }^{201} \mathrm{Tl}\right)$ are the two isotopes which are injected sequentially. ${ }^{99 \mathrm{~m}} \mathrm{Tc}$ delineates only the thyroid gland, whereas ${ }^{201} \mathrm{Tl}$ demonstrates both thyroid and the parathyroids. ${ }^{99 \mathrm{~m} T \mathrm{Tc}}$ thyroid image is then subtracted from the ${ }^{201} \mathrm{Tl}$ image, leaving behind ${ }^{201} \mathrm{Tl}$ avid regions that indicate parathyroid adenoma or hyperplasia.

Figure 1 for dual isotope subtraction technique.

\section{Washout technique}

This is based on the ability of the parathyroid adenoma to retain a tracer - Sestamibi or Myoview - both of which are commonly used for myocardial scanning. The 
early images show tracer uptake both in the parathyroid and the thyroid glands, whereas the delayed images (one hour and later) show tracer uptake only in the parathyroid.

Figure 2 for washout technique showing normal scan that is negative for parathyroid adenoma.

Figure 3 for washout technique showing abnormal scan that is positive for parathyroid adenoma on the left side involving the superior group.

Figure 4 showing abnormal scan that is positive for mediastinal parathyroid adenoma.

\section{Fusion imaging (CT+tomographic nuclear imaging)}

The lesions seen in nuclear imaging are not associated with any anatomical details thereby making it mandatory for additional imaging for anatomical localization pre-operatively. The recent innovation of fusing the anatomical CT images with nuclear imaging has overcome this technical deficiency and "the fusion imaging” has simplified pre-surgical imaging approach in many clinical areas including parathyroid adenoma.

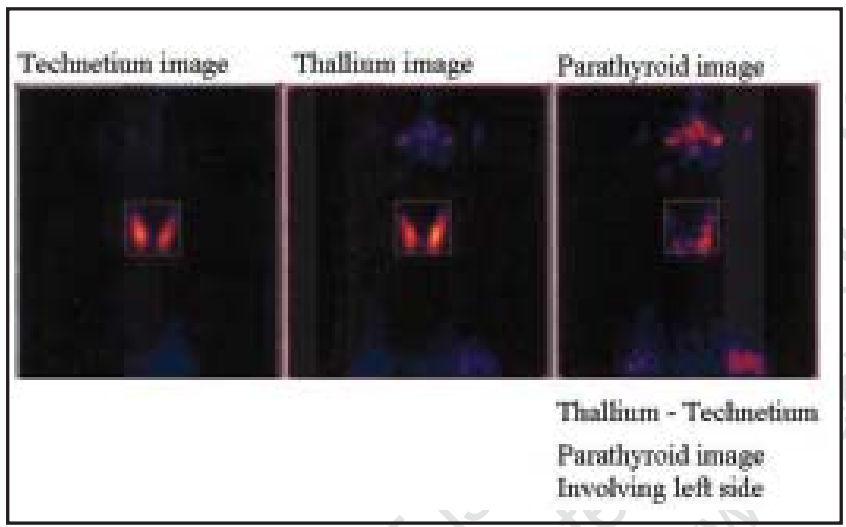

Figure 1: Dual isotope subtraction technique

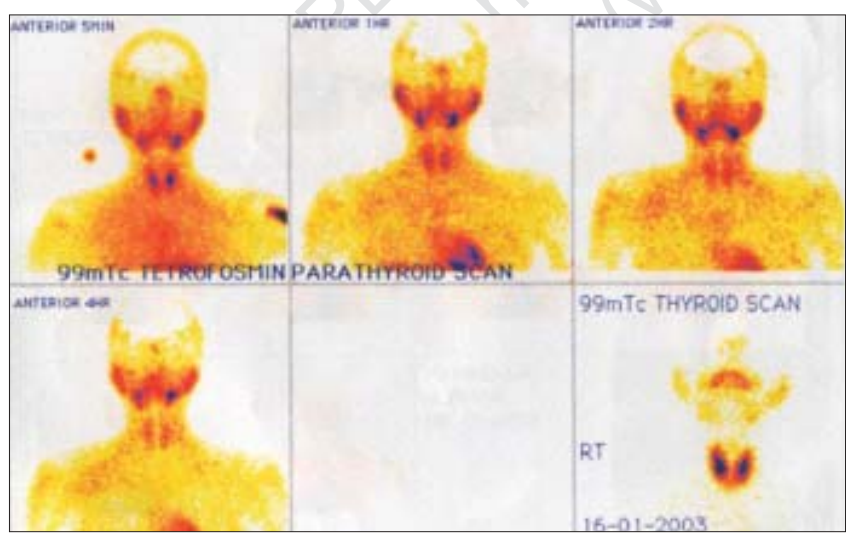

Figure 2: Washout technique showing normal scan that is negative for parathyroid adenoma
Figure 5 showing fusion image that is positive for parathyroid adenoma on the left side.

\section{Results}

The outcome of our study showed $57 \%$ true positives and $35 \%$ true negatives. These results were based on confirmation by the histopathology reports. Follow-up

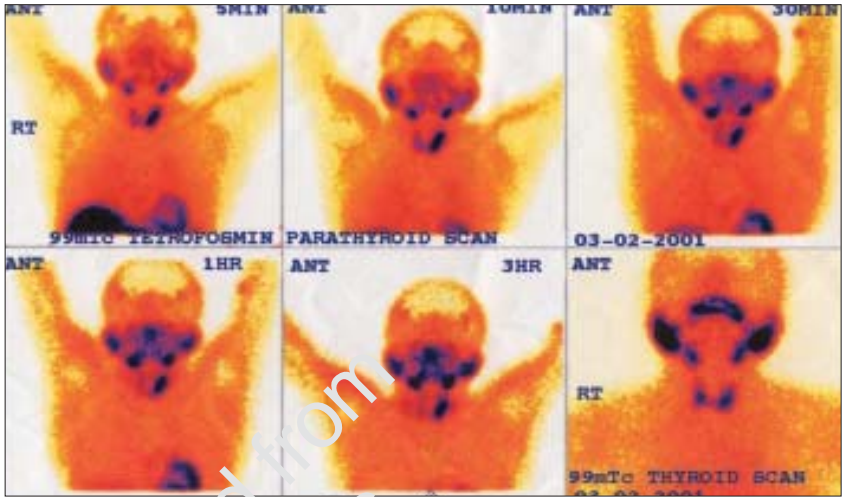

Figure 3: Washout technique showing abnormal scan that is positive for parathyroid adenoma on the left side involving the superior group.

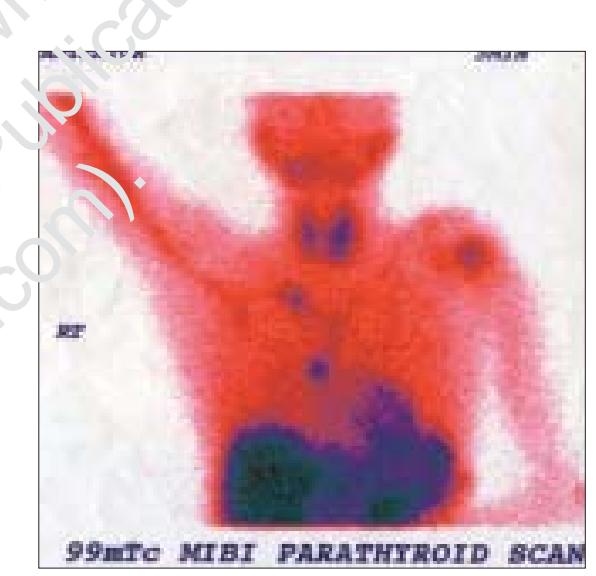

Figure 4: Showing abnormal scan that is positive for mediastinal parathyroid adenoma.

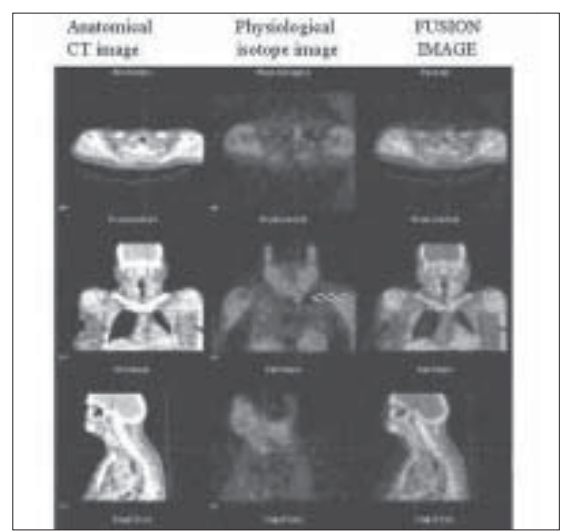

Figure 5: Showing fusion image that is positive for parathyroid adenoma on the left side. 
included browsing patients files within hospital record section and information from surgeons and patients themselves. There was one scan which was not conclusive, hence was grouped as an equivocal study. Our study showed an overall sensitivity of $94 \%$ and a specificity of $100 \%$ in the pre-operative detection of parathyroid adenomas.

The paper included 28 cases of suspected parathyroid adenoma studied with radionuclide scintigraphy. However, 28 cases studied with three different nuclear medicine techniques represent a group too small to conclude that "radionuclide scintigraphy should be the first choice of imaging modality in parathyroid adenoma". Though two techniques using two different isotopes have been included in this study, the use of SPECT and Fusion imaging are actually not additional methods, instead extension of the imaging procedures in both techniques. In this scenario, with the addition of fusion imaging, even with a study of 28 cases, radionuclide imaging would be the initial imaging modality of choice.

\section{Discussion}

The goal of treatment of hyperparathyroidism (most common cause being parathyroid adenoma) is to restore the patient to a normocalcemic euparathyroid state, to relieve symptoms and to prevent or possibly reverse complications of the disease. Resection of this parathyroid adenoma is the most effective treatment.

Accurate preoperative detection and localization of parathyroid adenomas is of utmost importance in patient care, not only in improving the outcome but also in shortening the duration of operation and thereby lowering the morbidity as well. The extent of the surgery is governed by the diagnosis of single versus multiple gland disease. Variation in parathyroid adenoma location (ectopic) may necessitate a different surgical approach or a more extensive exploration and is also one of the reasons for failure of the initial operation due to an unidentified ectopic location of the parathyroid adenoma. Successful minimally invasive parathyroidectomy ${ }^{[6]}$ is preferred treatment for primary hyperparathyroidism and is largely dependant on precise preoperative localization of the abnormal gland(s). Another situation where preoperative localization becomes crucial is reoperation for persistent or recurrent hyperparathyroidism which carries a higher risk of permanent hypocalcemia and recurrent laryngeal nerve injury than does primary exploration..$^{[7]}$

The various noninvasive imaging modalities other than radionuclide scintigraphy available for the preoperative detection and localization of parathyroid adenoma are ultrasonography, computed tomography and magnetic resonance imaging. ${ }^{[2,3,8]}$

With high resolution real time ultrasonography, $50 \%$ of the parathyroid adenomas can be accurately localized. ${ }^{[8,9]}$ As it is cheap and rapid, it is usually recommended as the initial investigation. However, cervical adenomas that cannot be located by ultrasonography tend to weigh less than $200 \mathrm{mg}$ and lie more medially behind the trachea or inferiorly behind the clavicular heads. Solitary low echogenic follicular thyroid adenoma or rare thyroid cyst can present a diagnostic problem in hyperparathyroidism. The overall success rate achieved by CT in a previously unexplored patient is $75 \%$. The use of thin section, dynamic contrast enhanced scans maximizes the chances of locating a parathyroid adenoma which shows as an enhancing nodule. CT is usually successful in the low cervical adenomas, but only $50 \%$ of the mediastinal adenomas can be detected. ${ }^{[8,10]}$ The sensitivity of MRI is greatest in the mediastinum, where ectopic abnormal glands are found (Sensitivity ranging from $50 \%-88 \%$ ) compared to $70 \%$ sensitivity in the neck. ${ }^{[8,10]}$ MRI can locate parathyroid tumors larger than $1 \mathrm{~cm}$ but can not distinguish small hyperplastic glands and though it performs better than CT but cannot precisely distinguish thyroid from adjacent parathyroid tumors.

Radionuclide scintigraphy demonstrates high sensitivity and specificity for parathyroid adenomas ${ }^{[3,11,13]}$ in the normal and ectopic locations, as well as in previously explored patients. ${ }^{[12]}$ Our study demonstrated a sensitivity of $94 \%$ and a specificity of $100 \%$ in the preoperative detection of parathyroid adenomas. Other studies in literature also demonstrate a sensitivity and specificity ranging between $60 \%-100 \% .^{[3,9,14-16]}$ This technology can be further extended for localization during operative procedures using the probe technology. Localization by radionuclide imaging prior to surgery, followed by using the probe per operatively, leads to more accurate surgical results.

From the common presentation of nephrolithiasis to the relatively lower prevalence of asymptomatic hyperparathyroidism, surgical treatment of the parathyroid adenoma is associated with low renal failure rate, low mortality and low morbidity (Russel et al). Hence, the current algorithm of imaging parathyroid adenoma would be: Sestamibi scan as the first procedure. If positive, surgical exploration. If equivocal, correlative MRI or CT is performed prior to surgery. If Sestamibi scan is negative, the diagnosis of parathyroid 
adenoma is not entertained.

\section{References}

1. Zwas ST, Czerniak A, Buchanan WS. The Parathyroids. Principles of Nucl Med 639,643-44,645.

2. Fraker DL. In: De Vita VT Jr, Hellman S, Rosenberg SA. Cancer: Principles and practice of oncology. $6^{\text {th }}$ ed. 2001. p. 1763.

3. Dijkstra B, Healy C, Kelly LM, McDermott EW, Hill AD, O'Higgins N. Parathyroid localization - current practice. JR Coll Edinb 2002;47:599-607.

4. O'Doherty MJ, Kettle AG, Wells P, Collins RE, Coakley AJ. Parathyroid imaging with ${ }^{99 \mathrm{~m} T C}$ - Sestamibi: Pre-operative localization and tissue uptake studies. J Nucl Med 1992;33:313-8.

5. Palestro CJ, Tomas MB, Tronco GG. Radionuclide imaging of the parathyroid glands. Semin Nucl Med 2005;35:266-76.

6. Norman J, Chheda H, Farrell C. Minimally invasive parathyroidectomy for primary hyperparathyroidism: Decreasing operative time and potential complications while improving cosmetic results. Am Surg 1998;64:391-6.

7. Neuman DR, Esselstyn CB, Eastwood J, Rice TW. Localization of mediastinal parathyroid adenoma in recurrent post operative hyperparathyroidism with ${ }^{99 \mathrm{mT}} \mathrm{Tc}$ - Sestamibi SPECT. Clin Nucl Med 1995;20: 175 .

8. Allison Grainger. 1308. Vol 2

9. Weiss MF, Beneke F, Dresel S, Schmid RA, Hahn K. Clinical impact of pre operative localization in patients presented with primary

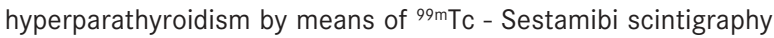
and ultrasound. JNM 2002;43:326-30.

10. Weber AL, Randolph G, Aksoy FG. The thyroid and parathyroid glands: CT and MR imaging and correlation with pathology and clinical findings. Radiol Clin North Am 2000;38:1105-29.

11. Saddadi F. Doubled-phase ${ }^{99 \mathrm{mT}} \mathrm{Tc}$ - Sestamibi imaging in localization of abnormal parathyroid glands. JNM 2002;43:125.

12. Vijayakumar V, Anderson ME. Detection of ectopic parathyroid adenoma by early ${ }^{99 \mathrm{~m} T c}$ sestamibi imaging. Ann Nucl Med 2005; 19: 157-9.

13. Taillefer R, Boucher Y, Potvin C, Lambert R. Detection and localization of parathyroid adenomas in patients with hyperparathyroidism using a single radionuclide imaging procedure with ${ }^{99 \mathrm{mT}} \mathrm{Tc}$ - Sestamibi (double - phase study). J Nucl Med 1992;33:1801-7.

14. Caixas A, Berna L, Hernandez A, Tebar FJ, Madariaga P, Vegazo $\mathrm{O}$, et al. Efficacy of preoperative diagnostic imaging localization of technetium ${ }^{99} \mathrm{~m}$-sestamibi scintigraphy in hyperparathyroidism. Surgery 1997; 121:535-41.

15. Diaz M, Boskisch A, Hahn K, Walgenbach S. Scintigraphic display

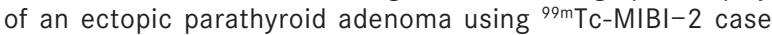
reports. Nuklearmedizin 1994;33:42-5.

16. Taillefer R. ${ }^{99 \mathrm{mT}} \mathrm{Tc}$ - Sestamibi parathyroid scintigraphy. In: Nuclear medicine annual. 1995. p. 51-75.

Source of Support: Nil, Conflict of Interest: None declared.

\section{Author Help: Reference checking facility}

The manuscript system (www.journalonweb.com) allows the authors to check and verify the accuracy and style of references. The tool checks the references with PubMed as per a predefined style. Authors are encouraged to use this facility before submitting articles to the journal.

- The style as well as bibliographic elements should be $100 \%$ accurate to get the references verified from the system. A single spelling error or addition of issue number / month of publication will lead to error to verifying the reference.

- Example of a correct style

Sheahan P, O'leary G, Lee G, Fitzgibbon J. Cystic cervical metastases: Incidence and diagnosis using fine needle aspiration biopsy. Otolaryngol Head Neck Surg 2002;127:294-8.

- Only the references from journals indexed in PubMed would be checked.

- Enter each reference in new line, without a serial number.

- Add up to a maximum 15 reference at time.

- If the reference is correct for its bibliographic elements and punctuations, it will be shown as CORRECT and a link to the correct article in PubMed will be given.

- If any of the bibliographic elements are missing, incorrect or extra (such as issue number), it will be shown as INCORRECT and link to possible articles in PubMed will be given. 